\title{
Leveraging budding yeast Saccharomyces cerevisiae for discovering aging modulation substances for functional food
}

\author{
Shaoyu Wang ${ }^{1 *}$ and Shujie Zhang ${ }^{1,2}$
}

${ }^{1}$ School of Community Health, Charles Sturt University, Orange, NSW 2800, Australia; ${ }^{2}$ College of Agronomy, China Agricultural University, Beijing 100193, China

Corresponding author: Shaoyu Wang, PhD, School of Community Health, Charles Sturt University, Orange, NSW 2800 Australia

Submission Date: October $25^{\text {th }}$, 2018. Acceptance Date: May $28^{\text {th }}$, 2019. Publication Date: May $30^{\text {st }}, 2019$.

Citation: Wang S., Zhang S. What can yeast tell us about aging modulation by bioactive substances in functional food. Functional Foods in Health and Disease 2019; 9(5): 297-311. DOI: https://doi.org/10.31989/ffhd.v9i5.575

\begin{abstract}
Functional food has been a practical mean to be used for health promotion and morbidity compression in the aging population. The discovery and development of bioactive substances with aging modulation actions are critical steps for more potent and safer functional food for aging modulation. Budding yeast (Saccharomyces cerevisiae), as a single-cell eukaryotic organism, displays genes, molecular and cellular mechanisms conserved with mammalians. It is a well-developed model for replicative and chronological aging in mammalians. This model organism, with its amenability by genetic and molecular tools, have thus served well for discovering bioactive compounds for aging modulation and health promotion of a variety of age-related health conditions in humans.

In this paper, we have, by synthesizing existing literature, summarized the characteristics of the budding yeast as a practical model for aging for mammalians including humans, then conceptualized a framework for discovering bioactive compounds using yeast cellular system for aging modulations for functional food research and industry. This framework consists of four components: uncovering targets for aging modulation, discovering and validating diet restriction mimetics, acting as cellular systems for screening natural products or synthetic compounds for aging modulation and being a biological factory for producing bioactive compounds according to the roles the yeast systems play. While these four components are continuously being harnessed in both research and industry, the last component of being a biological factory for producing bioactive compounds has largely unexplored, thus presenting a novel opportunity for the functional food industry.
\end{abstract}

Keywords: Aging modulation, budding yeast, functional food, bioactive substances, cell factory 


\section{INTRODUCTION:}

Life expectancy has increased dramatically during recent decades, partly due to the control of infectious disease and supply of enough food, coupled with a lower birth rate (at least in higher economic countries), leading to an ever-aging population. The consequence of this demographic trend is the rising of non-communicable diseases (NCDs) such as cancer, Alzheimer's disease and cardiovascular conditions [1]. Response to NCDs that is characterized by chronic nature and large scale, is not by speedy action with antibiotics rather requires ongoing and easy-to-implement actions. Treating or intervening aging has been proposed as the most effective strategy to prevent and slow down the onset of age-related diseases or NCDs, with the argument that aging processes are the most important cause of NCDs [2].

Food containing bioactive components, i.e. functional food (in a loose sense), in combination with other actions such as lifestyle change, is a realistic and scientifically proven approach to combat the aging processes with some successful outcomes [3]. Functional food is perhaps more important to the aged population than the younger ones (with no assumption of the aging processes not occurring in younger ages).

Discovery of bioactive substances naturally found in or to be added to the functional food, and revelation of the mechanism of their aging modulation capacity are imperative steps in developing better, safer, and more potent functional food for retarding aging and promoting health [3]. The bioactive compounds or substances in the functional food can be sourced from the plant-, animal-based materials and synthetics.

A variety of approaches can be used for discovering bioactive substances that modulate aging processes and extend a healthy life span. Animal models such as mice, flies, worms and single cell eukaryotic organism yeast have all greatly contributed to the discovery of bioactive substances for aging modulation $[4,5]$. Each of these models has its own unique features for investigating aging and its modulation. Yeast (Saccharomyces cerevisiae) as a eukaryotic model organism has been extensively used for studying replicative, chronological and colony aging, and is among the pioneer models for the discovery of Sirtuin genes [6-10]. Fundamental mechanisms of aging in yeast are found in mammals including humans, and worm (C. elegance) and insect (D. melanogaster) as well. Examples of the fundamental mechanisms are genome instability, dysfunction of mitochondria and stem cell exhaustion [11-13]. In this paper, we conceptualized four approaches using budding (or baker's) yeast to facilitate design, discovery and test of bioactive compounds for aging modulation to be used for the functional food industry.

\section{BEING A VITAL MODEL FOR AGING PROCESSES:}

Prior to describing the four approaches of the discovery of bioactive for functional food, we will first qualify how the budding yeast to be a suitable model for aging in humans. The aging phenotype defined as functional decline is conserved from yeast to humans. The course of aging in yeast is similar to insects, rodents and humans (Figure 1). A range of biological characteristics of the yeast makes this organism as an important aging model for more complex organisms including humans. (Table 1). Replicative aging and chronological aging phenotypes in budding yeast have been used to model aging of stem cells and post-mitotic cells, respectively, in the higher organisms including humans. 

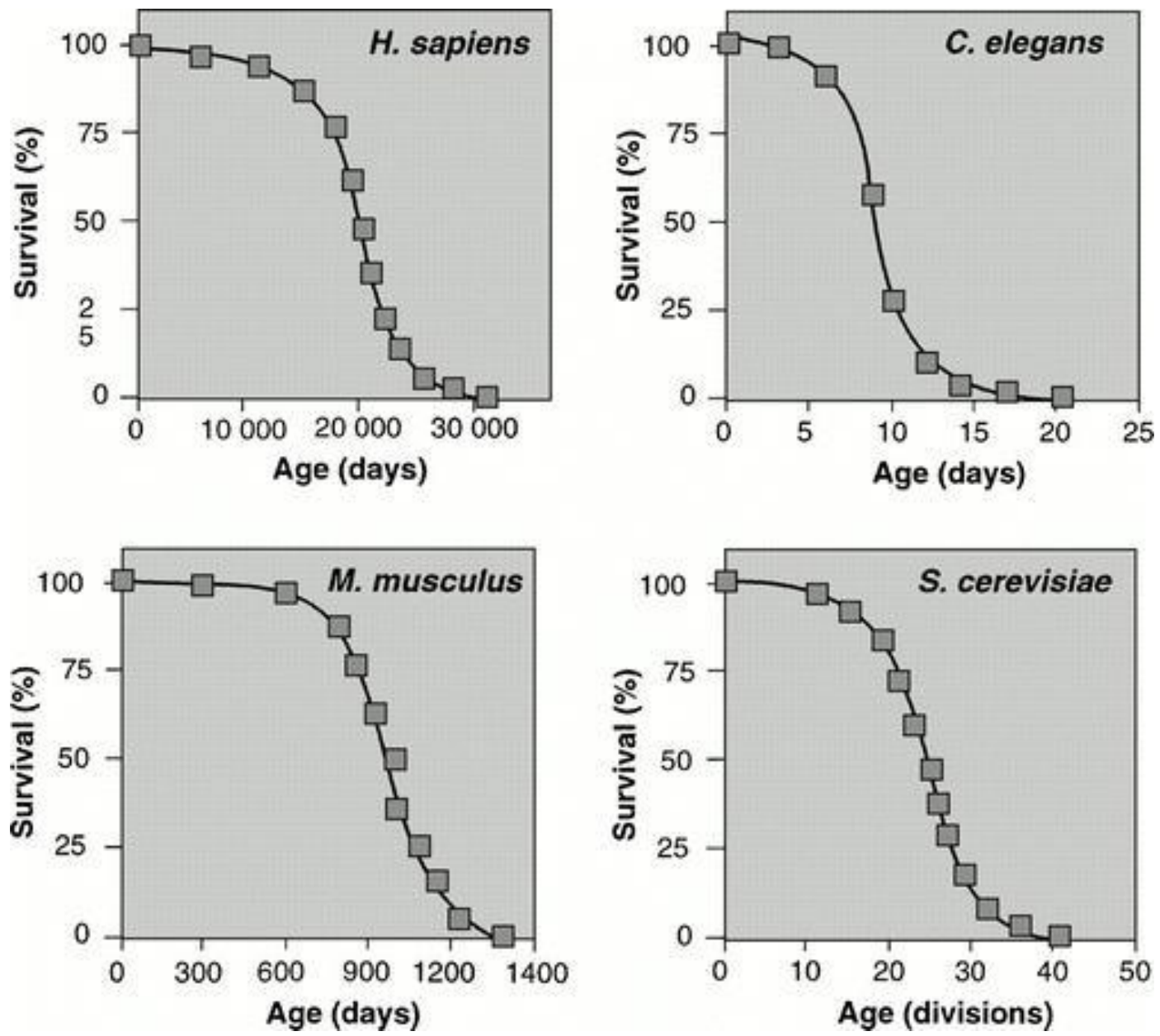

Figure 1 Aging is characterized as a functional decline of living units (cells, tissues, whole organisms) from yeast to humans.

[http://njms.rutgers.edu/departments/cell_biology_and_molecular_medicine/documents/Yeast_A ging_02_09_2015_Ivessa_MolecularMechanismsodDiseases.pdf]

The budding yeast displays the most hallmarks of aging in higher eukaryotes including humans [13]. For example, genomic instability [14], epigenetics [15] and proteostasis [16] have been empirically demonstrated in yeast. Additionally, its amenability by genetic, molecular biology and high-throughput tools makes this organism an invaluable tool for discovering bioactive substances that modulate aging processes (Table 1). This single cell organism has also contributed to the understanding of a very crucial question in aging research landscape: most eukaryotic organisms are aging, but why age-induced changes are not transmitted to the progeny? A seminal study on yeast has shown that resetting of lifespan occurs during gametogenesis, and transient induction of a transcription factor essential for later stages of gametogenesis extends the replicative lifespan of aged cells [17].

Considerable understanding of aging process and rejuvenation in yeast, conservation to humans and amenability with a range of biotechnological tools thus warrant this organism as a system for discovering bioactive substance for intervening aging including preventing, slowing and even reversing or rejuvenating aging, in addition to genetic approaches and dietary restriction [27, 28]. We conceptualised the four approaches using the yeast systems for achieving this purpose: discovering targets for aging modulation; uncovering and validating 
dietary restriction (DR) mimetics; acting as cellular systems for screening natural products or synthetic compounds for aging modulation and being a biological factory for producing bioactive compounds.

Table 1: Characteristics of yeast that qualify it as a model organism for discovering bioactive substances for modulating aging processes including those in humans.

\begin{tabular}{ll}
\hline Characteristics & Note \\
\hline $\begin{array}{l}\text { Small genome with 6000 genes; } \\
8 \text { genes in mitochondrial genome }\end{array}$ & $\begin{array}{l}18.5 \% \text { homologs and 40\% orthologs to humans [18]; At } \\
\text { least 47\% of genes can be humanised [19]; }\end{array}$ \\
$\begin{array}{l}\text { Replicative aging, chronological } \\
\text { aging and colony aging phenotypes }\end{array}$ & $\begin{array}{l}\text { Models for aging of mitotic cells (dividing), post-mitotic } \\
\text { tissues (non-dividing) and potentially multicellular tissue } \\
\text { in mammals }\end{array}$
\end{tabular}

Hallmarks of aging

Most hallmarks of aging in higher order organisms including human, such as genomic instability, exist in yeast [20]

Short life cycle e.g. less than 2 hours

Fast, robust and easy to work with and easy to culture

Comprehensive genetic, genomic, transcriptomic, proteomic and metabolomic data, and associated tools including bioinformatics*

Data and systems biology analysis of findings for understanding the mechanisms of aging and its modulation by bioactive compounds and translate the discovery to higher organisms and humans

Cell based high-throughput technical tools

Suitable for screening for aging modulation compounds individually, in combination or crude extracts from plants [21-25], also enabling to variation of aging phenotypes at an individual cells level [26].

This is in sharp contrast to in vitro non-cell based approaches

* Note: powerful genetics, proteomics, completely sequenced genome, comprehensive single gene deletion and overexpression libraries, determination of protein localization in vivo and tandem affinity purification-tagging approaches and synthetics biology; Saccharomyces Genome Database (SGD) - https://www.yeastgenome.org/ and other bioinformatics tools.

\section{DISCOVERING TARGETS FOR AGING MODULATION:}

Budding yeast provides powerful single cell systems to discover or validate genes, molecular and cellular pathways of aging. This organism displays the most of known hallmarks of aging relevant in higher eukaryotes including humans [13]. For example, genomic instability [14], epigenetics [15] and proteostasis [29] have been empirically demonstrated in yeast. An important pathway that is closely relevant to functional food is the nutrient sensing and regulation pathway - Insulin/IGF-I and related signalling pathways (Figure 2). Cell membrane receptors and intracellular cascade of signalling pathways and till the gene expressions via transcriptional factors are shown to be remarkably conserved among these organisms. These genes, proteins and components of the pathways of the aging hallmarks can be the targets for discovering and developing compounds for modulating aging including preventing, delaying and reversing aging, and treating aging-related diseases [14, 15, 30, 31]. 




Figure 2: The conservation of nutrients sensing and regulation pathways from yeast to rodents [32].

The recent discovery of yeast protein kinase Sch9 that adjusts V-ATPase assembly/disassembly to control $\mathrm{pH}$ homeostasis and longevity in response to glucose availability may be proven to be a useful target in discovering bioactive for aging modulation [33]. A 2017 study has pushed the frontier further. Pol III was discovered as a pivotal output of the target of rapamycin (TOR) nutrient signalling network for longevity, and the evolutionary conservation of Pol III warrants its potential as a therapeutic target [34]. Sir2 family of genes and proteins have been the well-known targets for developing aging modulating compounds which were discovered initially in the budding yeast [35]. The yeast silent information regulator 2 (SIR2) gene, which conferred sirtuins their family name, was first shown to extend lifespan in yeast two decades ago. The sirtuin proteins are NAD ${ }^{+}$ dependent protein deacetylases that serve as regulatory functions through deacetylation of histone and a wide range of other proteins. As Bonkowski and Sinclair [31] noted, more than 14,000 sirtuin-activating compounds (STACs) have been synthesized thus far, and a handful have been subjected to be tested in animal models of type 2 diabetes, colitis, neurodegeneration, fatty liver and atherosclerosis, among others [36]. STACs now are in their fifth generation and some have more than 1,000-fold greater potency in vitro than resveratrol. The STAC named SRT2104, which mimics aspects of calorie restriction and extends male mouse lifespan, has advanced through multiple phase I trials with few, if any, side effects to phase II trials. Two SRT2104 clinical trials in elderly volunteers and otherwise healthy smokers showed a slight reduction in body weight, a 15-30\% improvement in the cholesterol ratio and a $19 \%$ decrease 
in triglyceride levels [36] (Table 2). The resveratrol is, however, still the best known sirtuins activator and importantly it is present in various diet sources such as grape skin, apple and peanuts [37, 38] and it is well into the clinical trials [37].

Table 2: Examples of compounds that stimulate sirtuins directly or via $\mathrm{NAD}^{+}$for aging modulation.

\begin{tabular}{lll}
\hline Compounds & Action & Source/Note \\
\hline $\begin{array}{l}\text { Resveratrol: a polyphenolic } \\
\text { compound (Phases III-IV clinical } \\
\text { trails) }\end{array}$ & $\begin{array}{l}\text { Direct activator; the most } \\
\text { potent activator }\end{array}$ & Grape, peanuts, apple, \\
$\begin{array}{l}\text { quercetin and apigenin } \\
\text { (flavonoids) }\end{array}$ & $\begin{array}{l}\text { Boost NAD }{ }^{+} \text {via inhibitor of } \\
\text { CD38 }\end{array}$ & Fruits and vegetables [39-41] \\
$\begin{array}{l}\text { nicotinamide mononucleotide } \\
\text { (NMN) }\end{array}$ & Boost NAD ${ }^{+}$directly & Fruits and vegetables \\
$\begin{array}{l}\text { nicotinamide riboside (NR) } \\
\text { (Phase II clinical trials) }\end{array}$ & & \\
$>14,000$ synthetic STACs, e.g. & Direct activators via & Synthetics [36] \\
$\begin{array}{l}\text { SRT1720 ( Imidazothiazoles), } \\
\text { STAC-2 (Hiazolopyridines), }\end{array}$ & allosteric activation. & \\
$\begin{array}{l}\text { STAC-5 (Benzimidazoles), } \\
\text { SRT2104 (Phase II trials) }\end{array}$ & & \\
\hline
\end{tabular}

Note: STACs: sirtuin-activating compounds

It is very exciting to see the target driven approach in working to discover the bioactive compounds for functional food. However, it must be kept in mind that it is necessary that animal tests, clinical trials, and epidemiology studies need to be carried out for their other properties needed for the treatment of age-related diseases. A 9 years follow-up study (outcomes at $3^{\text {rd }}, 6^{\text {th }}$ and $9^{\text {th }}$ year) of resveratrol as part of the diet of 769 participants aged 65 years and older, showed the inverse relationship between concentrations of diet and urinary resveratrol and frailty syndrome, supporting that the resveratrol can be effective in preventing certain age-related diseases in humans. However, limited high-quality clinical trials of resveratrol showed that more trials are still needed, particularly in regard to its bioavailability and in varied populations [42].

The budding yeast is also an excellent model for understanding aging-related diseases such as neurological diseases in humans [19]. Human genes for particular age-related diseases have been successfully incorporated into yeast genome and have been expressed [43, 44]. The human genes and their products can then be used as targets in yeast systems for discovering bioactive compounds including those in functional food. For example, human $\beta$-secretase that plays an important role in Alzheimer's disease (AD) pathology has been expressed in yeast and its inhibitors have been subsequently identified [45, 46]. Middendorp et al. [46] have used the yeast system that expressed human $\beta$-secretase to validate the BACE- 1 inhibitors as target specific compounds. This approach is applicable in high-throughput screens for selecting inhibitors of defined secretases acting on natural substrates in a membrane-bound protein configuration. Promising phytochemicals indicated from various means [47] can be subjected to high-throughput screening for their potential for AD prevention or therapeutic agents [22]. 
Another relevant example of yeast aging pathways as targets for humans aging is the autophagy, which is a recycling pathway within cells that delays aging via eliminating injured organelles and other molecules [48-50]. The pathway and longevity in yeast have been shown to be positively modulated by a bioactive, the ethanolamine which is a precursor of phosphatidylethanolamine [50]. This chemical is widely used in cosmetics, lifestyle, and household products such as hair colours, washing powders, and so forth $[50,51]$ which is reported to be tolerated by human skin. Whether this chemical can be fortified in functional food, and the efficacy and safety of ethanolamine-containing food remains as an interest to pursue. It is noted here, that compounds that targeting autophagy for aging modulation are largely unexplored [52]. Possible targets for aging modulation may also come from the metabolomics studies. Various methods for metabolite profiling at single-cell, few-cells and population levels and during aging process of yeast have been developed [53, 54].

The accumulating evidence suggests that yeast continues to be an excellent system for discovering targets for aging modulation, treatment of diseases of aging, and beyond for other human diseases such as diabetes mellitus due to its capacity to be humanized by various approaches.

\section{DISCOVERING AND VALIDATING DIET RESTRICTION MIMETICS:}

Dietary restriction (DR) defined as the food restriction without malnutrition is a major, scientifically proven mean for aging modulation and health promotion from yeast to nonhuman mammals [55-57], and evidence is emerging for humans too [58]. However, its application to humans is proven to be difficult, due to psychology and lifestyle issues. In response to these issues, DR mimetics known as drugs that mimic beneficial effects of dietary restriction without malnutrition, are being developed [59]. DR in present paper refers specifically to caloric restriction and amino acids restriction. Considerable understanding of pathways underlying DR in regulating yeast longevity and aging has been made, including Snf1 (AMPK- AMP-activated protein kinase) signalling pathway [60], TOR (target of rapamycin) pathway [61], Sir2 pathway [62] and cell cycle program [63]. While SIR2 pathway is responsive to caloric restriction, the TOR pathway is for amino acids sensing [61].

The identification and validation of DR mimetics using yeast as a model have been explored, particularly due to the close link of DR to nutrient sensing pathways $[64,65]$. The resveratrol, a plant polyphenol was first identified as DR-mimetics in yeast, acting on sirtuins pathway by 'monitoring' low-energy states via detecting high $\mathrm{NAD}^{+}$levels. Resveratrol elicits physiological changes mimicking DR, leading to improved tissue pathology, increased endurance, mitochondrial biogenesis, energy expenditure, insulin sensitivity, and decreased fat accumulation, blood insulin and IGF-1 in higher eukaryotes [66, 67]. Compounds that raise $\mathrm{NAD}^{+}$levels, such as nicotinamide riboside and nicotinamide mononucleotide (NMN) as described above show a great promise as DR-mimetics to treat numerous age-related conditions in humans.

Activators, both synthetics and natural compounds, of AMPK pathway have also been identified. More than 100 natural products or extracts derived from plants including those for Asian medicines have been demonstrated to activate AMPK [68]. For example, berberine [69, 70], and arctigenin [71] seem to activate AMPK by inhibiting the mitochondrial respiratory chain and galegine, a natural product from the medicinal plant Galega officinalis from which metformin and phenformin were derived [72]. More details on these natural compounds can be 
found in the paper by Hardie [68]. As the mechanisms of actions of most of these compounds have not been explored, yeast, with its advantageous features for aging modulation, can be leveraged for elucidating such mechanisms and qualifying their capacity as DR-mimetics as parts of functional food. Relevance of AMPK pathway and subsequent mimetics to human diseases of aging renders studies on this pathway as an emerging research area [73]. However, it is useful to be reminded that AMPK pathway can control intrinsic aging in yeast independent of DR [74]. Other known or potential DR-mimetics including polyphenols (such as quercetin, anthocyanin, and curcumin), lactoferrin, lutein, and eicosapentaenoic acid may be investigated at molecular levels using yeast system for their efficacy and mechanisms of action in aging modulation in yeast [75].

\section{ACTING AS CELLULAR SYSTEMS FOR SCREENING NATURAL PRODUCTS OR SYNTHETIC COMPOUNDS FOR AGING MODULATION:}

Single cell eukaryote budding yeast displays three aging phonotypes: replicative, chronological and colony aging which represents the three states of cells in multicellular organisms including humans, i.e. models for aging of mitotic cells including stem cells (dividing), post-mitotic tissues (non-dividing, i.e. functioning cells) and potentially multicellular tissue in mammals (Table 1). The strongest advantage of this organism is that it is easily amendable to various methods of high throughput screening assays. This is nearly unmatched by any other model organisms for an aging study [21, 22, 24, 27].

The replicative aging phenotypes have been assessed by various screening tools. The limiting factor in studying this phenotype is the difficulty in separating daughter cells from mother cells. The pioneering work by Mortimer and Johnston in 1959, used micromanipulation physically to remove daughter cells from mothers under the microscope [76]. However, this assay is not easy to perform and is labour intensive although it remains as the standard method. Particularly, it is not amendable to large scale screening assay. Various means are then developed to separate the mothers from daughters for high-throughput assays. Using genetic methods, the mothers can be enriched, where the daughter cells are genetically eliminated. This genetic methods provide a simplified screening method for identifying genetic or environmental factors that regulate replicative aging [77]. Taking the advantage of size difference between mother and buds, physical separation of mothers and daughter have been made using microfluidics and mechanical principals $[24,78]$. Separation of mother cells from daughter cells have been made by leveraging the knowledge of the previously synthesized components of the mother cell surface do not contribute to the daughter cell surface. A biotinavidin system has been used to harness knowledge in which mother cells are labelled with biotin and then grown for desired number of generations. The labelled mothers in the culture are then extracted using a streptavidin-coated magnetic beads [79].

The chronological aging has also been subjected to a high throughput screen with the outgrowth assay as the readout. A high-throughput method for measuring chronological aging involves first growing yeast cultures to stationary phase in individual wells of a 96-well plate. At various intervals (time points), a sample (e.g. $1 \mu \mathrm{L}$ ) from each well is transferred into a corresponding well of a second 96-well plate containing the usual amount (e.g. $200 \mu \mathrm{L}$ ) of fresh 
media. This second plate is incubated for a short period (e.g. $24 \mathrm{~h}$ ) at a constant temperature, after which the optical density (OD) at $600 \mathrm{~nm}$ of each well is determined using a plate-reader. This OD corresponds to the number of viable cells in the inoculum [80, 81].

Yeast cells obtained in these two types of high-throughput assays of aging have been exploited for discovering anti-aging compounds. Lithocholic acid as an anti-aging compound has been identified as being able to extend yeast chronological life span in a TOR-independent manner [81]. Another example is that, in 2014, glyceollin I, an induced phytoalexin in soybeans were discovered. It extends yeast life span at low (nM) doses in a calorie restriction (CR)dependent manner. The glyceollin I might be a promising DR-mimetic candidate, and the high contents of glyceollins in soybeans could improve its bioactivity as functional food ingredients [82]. The same year, yeast was also used for screening more than 100 botanical materials. This screen identified tanshinones that extend chronological lifespan up to 2.5 times compared with control [83].

It is anticipated that continued screening of natural products using various aging assays, coupled with ever developed new tools for modulating aging processes [27] will be conducted, especially in the context of seemingly abundant amount of natural materials and the quest for more potent, safer medicine and functional food for better health via aging modulation.

\section{BEING A BIOLOGICAL FACTORY FOR PRODUCING BIOACTIVE COMPOUNDS:}

Yeast can also serve as a biological factory for producing bioactive compounds for modulating aging $[84,85]$. The rationale for this approach is that direct extraction of bioactive compounds from natural biological sources can be inefficient, due to the low concentrations of these compounds, and their chemical synthesis is often difficult to achieve because of their complex structures. In recent years, the discovery of genes involved in the synthetic pathways of the metabolites, combined with synthetic biology tools, has allowed the construction of yeast cell factories for the production of these metabolites from renewable biomass [86].

There are several characteristics making yeast as cell factories for bioactive compounds production. First, complete synthetic pathways can be assembled in a single step including subcellular pathways engineering [87-91]. For example, a gene cluster that contains four plantderived genes of the early flavonoid biosynthetic pathway is constructed to allow the conversion of phenylpropanoid acids into flavanones in budding yeast [89]. Second, the yeast is amendable using the latest gene editing tool the CRISPRCas9 technology. For example, the mevalonate pathway created using this technology in yeast, has produced the mevalonate titers greater than 41-fold compared to the wild-type strain [92]. This technology has not been used widely in aging modulation research yet at least in term of production of important natural bioactive compounds in large quantity and more economical manner. Other examples are the flavonoids, stilbenoids (e.g. quercetin, kaempferol and resveratrol), ginsenoside and melatonin produced using this approach [84, 93-95]. However, the public perception and acceptance of the genetically modified health promotion medicine or substance can be an issue. This approach nevertheless is an excellent tool for producing bioactive aging modulation compounds is clearly largely underexplored. 


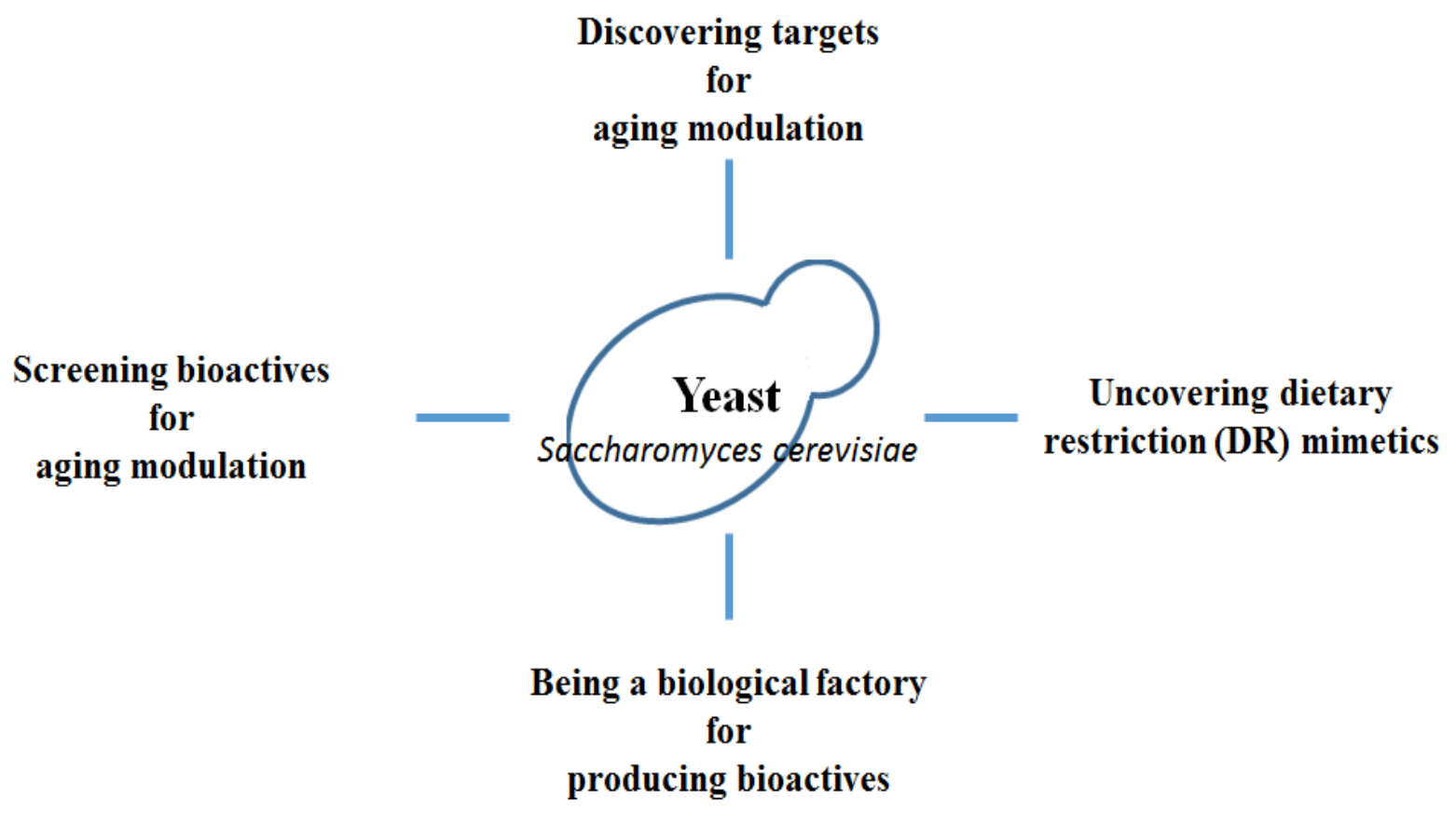

Figure 3 Four approaches using Yeast (Saccharomyces cerevisiae) system for functional food have been developed.

\section{CONCLUSIONS}

In this paper, we argue that four approaches exist to produce effective medicinal products for aging modulations that are applicable to humans. The powerful approach of yeast being the cell factories for aging modulation research has unfortunately been largely unexplored, but the opportunity is clear. With these approaches in mind, it is conceivable that efficiently quantifying aging modulating effects of functional food for health is becoming practical. The knowledge gained in using these approaches will greatly facilitate pre-clinical development of ever better medicine for prevention, slowing and treating of aging and diseases of aging in this ever-aging population.

Abbreviations: DR: Diet restriction encompasses both of caloric restriction (CR) and diet restriction (DR) of nutrients such as amino acids. Other common abbreviations include Alzheimer's disease (AD); sirtuin-activating compounds (STACs); target of rapamycin (TOR); nicotinamide mononucleotide (NMN).

Competing Interests: The authors have no financial interests or any other conflicts of interest to disclose.

Authors' Contributions: SW conceived and wrote the first draft. SW and SZ contributed to the revision of the text. The authors agreed to publish in this journal.

Acknowledgements and Funding: This work was funded by Agricultural Education and Research Grant from Charles Sturt University. Authors are grateful for this support from the University 


\section{REFERENCES}

1. Bongaarts, J.: Human population growth and the demographic transition. Philosophical Transactions of the Royal Society B: Biological Sciences, 2009; 364(1532): p. 29852990.

2. Fontana, L., B.K. Kennedy, V.D. Longo: Treating Ageing. Nature, 2014; 511: p. 405407.

3. Hasler, C.M., Functional Foods: Benefits, Concerns and Challenges - A Position Paper from the American Council on Science and Health. The Journal of Nutrition, 2002; 132(12): p. 3772-3781.

4. Xiang, L., et al.: Anti-aging effects of phloridzin, an apple polyphenol, on yeast via the SOD and Sir2 genes. Biosci Biotechnol Biochem, 2011; 75(5): p. 854-8.

5. Aruoma, O.I., et al.: Applications and bioefficacy of the functional food supplement fermented papaya preparation. Toxicology, 2010; 278(1): p. 6-16.

6. Guarente, L.: Sirtuins in aging and disease. Cold Spring Harb Symp Quant Biol, 2007; 72: p. 483-8.

7. MacLean, M., N. Harris, P.W. Piper: Chronological lifespan of stationary phase yeast cells; a model for investigating the factors that might influence the ageing of postmitotic tissues in higher organisms. Yeast, 2001; 18(6): p. 499-509.

8. Longo, V.D., et al.: Replicative and Chronological Aging in Saccharomyces cerevisiae. Cell Metabolism, 2012; 16(1): p. 18-31.

9. Carmona-Gutierrez, D. S. Büttner: The many ways to age for a single yeast cell. Yeast (Chichester, England), 2014; 31(8): p. 289-298.

10. Vachova, L., M. Cap, Z. Palkova: Yeast colonies: a model for studies of aging, environmental adaptation, and longevity. Oxid Med Cell Longev, 2012; p. 601836.

11. Longo, V.D. P. Fabrizio: Regulation of longevity and stress resistance: a molecular strategy conserved from yeast to humans? Cell Mol Life Sci, 2002; 59(6): p. 903-8.

12. Wasko, B.M., M. Kaeberlein: Yeast replicative aging: a paradigm for defining conserved longevity interventions. FEMS Yeast Res, 2014; 14(1): p. 148-59.

13. Lopez-Otin, C., et al.: The hallmarks of aging. Cell, 2013. 153(6): p. 1194-217.

14. Sinclair, D.A., L. Guarente: Extrachromosomal rDNA circles--a cause of aging in yeast. Cell, 1997; 91(7): p. 1033-42.

15. Kaeberlein, M., M. McVey, L. Guarente: The SIR2/3/4 complex and SIR2 alone promote longevity in Saccharomyces cerevisiae by two different mechanisms. Genes Dev, 1999; 13(19): p. 2570-80.

16. Janssens, G.E., et al.: Protein biogenesis machinery is a driver of replicative aging in yeast. Elife, 2015; 4: p. e08527.

17.Unal, E., B. Kinde, A. Amon: Gametogenesis eliminates age-induced cellular damage and resets lifespan in yeast. Science 2011; 332(6037): p. 1554-1557.

18. Karathia, H., et al.: Saccharomyces cerevisiae as a model organism: a comparative study. PLoS One, 2011; 6(2): p. e16015.

19. Kachroo, A.H., et al.: Systematic humanization of yeast genes reveals conserved functions and genetic modularity. Science, 2015; 348(6237): p. 921-5.

20. Janssens, G.E., L.M: Veenhoff, Evidence for the hallmarks of human aging in replicatively aging yeast. Microb Cell, 2016; 3(7): p. 263-274.

21. Tucker, C.L.: High-throughput cell-based assays in yeast. Drug Discov Today, 2002; 7(18 Suppl): p. S125-30. 
22. Sarnoski, E.A., P. Liu, M. Acar, A: High-Throughput Screen for Yeast Replicative Lifespan Identifies Lifespan-Extending Compounds. Cell reports, 2017; 21(9): p. 26392646.

23. Jung, P.P., et al.: Protocols and programs for high-throughput growth and aging phenotyping in yeast. PLoS One, 2015; 10(3): p. e0119807.

24. Jo, M.C., et al.: High-throughput analysis of yeast replicative aging using a microfluidic system. Proc Natl Acad Sci U S A, 2015; 112(30): p. 9364-9.

25. Jo, M.C., L. Qin: Microfluidic Platforms for Yeast-Based Aging Studies. Small (Weinheim an der Bergstrasse, Germany), 2016; 12(42): p. 5787-5801.

26. Smith, J.T., et al.,: Yeast lifespan variation correlates with cell growth and SIR2 expression. PLoS One, 2018; 13(7): p. e0200275.

27. Zimmermann, A., et al.: Yeast as a tool to identify anti-aging compounds. FEMS Yeast Research, 2018; p. foy020-foy020.

28. Katewa, S.D., P. Kapahi: Dietary restriction and aging, 2009; Aging cell, 2010. 9(2): p. 105-112.

29. Janssens, G.E., et al.: Protein biogenesis machinery is a driver of replicative aging in yeast. eLife, 2015; 4: p. e08527-e08527.

30. Grabowska, W., E. Sikora, A. Bielak-Zmijewska: Sirtuins, a promising target in slowing down the ageing process. Biogerontology, 2017; 18(4): p. 447-476.

31. Kennedy, B.K., et al.: Mutation in the silencing gene SIR4 can delay aging in S. cerevisiae. Cell, 1995; 80(3): p. 485-96.

32. Parrella, E., V.D. Longo: Insulin/IGF-I and related signaling pathways regulate aging in nondividing cells: from yeast to the mammalian brain. ScientificWorldJournal, 2010; 10: p. 161-77.

33. Wilms, T., E. Swinnen, E. Eskes: The yeast protein kinase Sch9 adjusts V-ATPase assembly/disassembly to control $\mathrm{pH}$ homeostasis and longevity in response to glucose availability. 2017; 13(6): p. e1006835.

34. Filer, D., et al.: RNA polymerase III limits longevity downstream of TORC1. Nature, 2017; 552(7684): p. 263-267.

35. Dai, H., et al.: Synthesis and Assay of SIRT1-Activating Compounds. Methods in enzymology, 2016; 574: p. 213-244.

36. Bonkowski, M.S., D.A.: Sinclair, Slowing ageing by design: the rise of NAD(+) and sirtuin-activating compounds. Nature reviews. Molecular cell biology, 2016; 17(11): p. 679-690.

37. Berman, A.Y., et al.: The therapeutic potential of resveratrol: a review of clinical trials. npj Precision Oncology, 2017; 1(1): p. 35.

38. Weiskirchen, S., R. Weiskirchen, Resveratrol: How Much Wine Do You Have to Drink to Stay Healthy? Advances in Nutrition, 2016; 7(4): p. 706-718.

39. Davis, J.M., E.A. Murphy, M.D. Carmichael: Effects of the dietary flavonoid quercetin upon performance and health. Curr Sports Med Rep, 2009; 8(4): p. 206-13.

40. Yan, X., et al.: Apigenin in cancer therapy: anti-cancer effects and mechanisms of action. 2017; 7: p. 50.

41. Chung, S., et al.: Regulation of SIRT1 in cellular functions: role of polyphenols. Archives of biochemistry and biophysics, 2010; 501(1): p. 79-90.

42. Tome-Carneiro, J., et al.: Resveratrol and clinical trials: the crossroad from in vitro studies to human evidence. Curr Pharm Des, 2013; 19(34): p. 6064-93. 
43. Bassett, D.E., Jr., M.S. Boguski, P. Hieter: Yeast genes and human disease. Nature, 1996; 379(6566): p. 589-90.

44. Bharadwaj, P., R. Martins, I. Macreadie: Yeast as a model for studying Alzheimer's disease. FEMS Yeast Res, 2010; 10(8): p. 961-9.

45. Luthi, U., et al.: Human beta-secretase activity in yeast detected by a novel cellular growth selection system. Biochim Biophys Acta, 2003; 1620(1-3): p. 167-78.

46. Middendorp, O., et al.: Yeast growth selection system for the identification of cell-active inhibitors of beta-secretase. Biochim Biophys Acta, 2004; 1674(1): p. 29-39.

47. Kumar, N.S., N. Nisha: Phytomedicines as potential inhibitors of $\beta$ amyloid aggregation: significance to Alzheimer's disease. Chinese Journal of Natural Medicines, 2014; 12(11): p. 801-818.

48. Tyler, J.K., J.E. Johnson: The role of autophagy in the regulation of yeast life span. Ann N Y Acad Sci, 2018; 1418(1): p. 31-43.

49. Abeliovich, H.: Regulation of autophagy by amino acid availability in S. cerevisiae and mammalian cells. Amino Acids, 2015; 47(10): p. 2165-75.

50. Rockenfeller, P., et al.: Ethanolamine: A novel anti-aging agent. Molecular and cellular oncology, 2015; 3(1): p. e1019023-e1019023.

51. Rockenfeller, P., et al.: Phosphatidylethanolamine positively regulates autophagy and longevity. Cell Death Differ, 2015; 22(3): p. 499-508.

52. Galluzzi, L., et al.: Pharmacological modulation of autophagy: therapeutic potential and persisting obstacles. Nature Reviews Drug Discovery, 2017; 16: p. 487.

53. Richard, V.R., S.D. Bourque, and V.I. Titorenko: Metabolomic and lipidomic analyses of chronologically aging yeast. Methods Mol Biol, 2014; 1205: p. 359-73.

54. Ibáñez, A.J., et al.: Mass spectrometry-based metabolomics of single yeast cells. Proceedings of the National Academy of Sciences, 2013; 110(22): p. 8790-8794.

55. Taormina, G., M.G. Mirisola: Calorie restriction in mammals and simple model organisms. Biomed Res Int, 2014; p. 308690.

56. Fontana, L., L. Partridge: Promoting Health and Longevity through Diet: From Model Organisms to Humans. Cell, 2015; 161(1): p. 106-118.

57. McCay, C.M., M.F. Crowell, L.A. Maynard: The effect of retarded growth upon the length of life span and upon the ultimate body size. 1935. Nutrition, 1989; 5(3): p. 15571; discussion 172.

58. Redman, L.M., et al.: Metabolic Slowing and Reduced Oxidative Damage with Sustained Caloric Restriction Support the Rate of Living and Oxidative Damage Theories of Aging. Cell Metab, 2018; 27(4): p. 805-815.e4.

59. Schleit, J., B.M. Wasko,M. Kaeberlein: Yeast as a model to understand the interaction between genotype and the response to calorie restriction. FEBS letters, 2012; 586(18): p. 2868-2873.

60. Wierman, M.B., et al: Caloric Restriction Extends Yeast Chronological Life Span by Optimizing the Snf1 (AMPK) Signaling Pathway. Mol Cell Biol, 2017; 37(13).

61. Dann, S.G., G. Thomas: The amino acid sensitive TOR pathway from yeast to mammals. FEBS Letters, 2006; 580(12): p. 2821-2829.

62. Dang, W.: The controversial world of sirtuins. Drug discovery today. Technologies, 2014; 12: p. e9-e17.

63. Leonov, A., et al.: Caloric restriction extends yeast chronological lifespan via a mechanism linking cellular aging to cell cycle regulation, maintenance of a quiescent 
state, entry into a non-quiescent state and survival in the non-quiescent state. Oncotarget, 2017; 8(41): p. 69328-69350.

64. Kyryakov, P., et al.: Caloric restriction extends yeast chronological lifespan by altering a pattern of age-related changes in trehalose concentration. Front Physiol, 2012; 3: p. 256.

65. Lane, M.A., G.S. Roth, D.K. Ingram: Caloric restriction mimetics: a novel approach for biogerontology. Methods Mol Biol, 2007; 371: p. 143-9.

66. Chen, D., L. Guarente: SIR2: a potential target for calorie restriction mimetics. Trends Mol Med, 2007; 13(2): p. 64-71.

67. Baur, J.A., et al.: Resveratrol improves health and survival of mice on a high-calorie diet. Nature, 2006; 444(7117): p. 337-42.

68. Grahame Hardie, D.: Regulation of AMP-activated protein kinase by natural and synthetic activators. Acta Pharm Sin B, 2016; 6(1): p. 1-19.

69. Hawley, S.A., et al.: Use of Cells Expressing $\gamma$ Subunit Variants to Identify Diverse Mechanisms of AMPK Activation. Cell Metabolism, 2010; 11(6): p. 554-565.

70. Turner, N., et al.: Berberine and its more biologically available derivative, dihydroberberine, inhibit mitochondrial respiratory complex I: a mechanism for the action of berberine to activate AMP-activated protein kinase and improve insulin action. Diabetes, 2008; 57(5): p. 1414-8.

71. Huang, S.L., et al.: Arctigenin, a natural compound, activates AMP-activated protein kinase via inhibition of mitochondria complex I and ameliorates metabolic disorders in ob/ob mice. Diabetologia, 2012; 55(5): p. 1469-81.

72. Mooney, M.H., et al.: Mechanisms underlying the metabolic actions of galegine that contribute to weight loss in mice. Br J Pharmacol, 2008; 153(8): p. 1669-77.

73. Li, J., et al.: Dissecting the role of AMP-activated protein kinase in human diseases. Acta Pharmaceutica Sinica B, 2017; 7(3): p. 249-259.

74. Lu, J.-Y., et al.: Acetylation of yeast AMPK controls intrinsic aging independently of caloric restriction. Cell, 2011; 146(6): p. 969-979.

75. Gillespie, Z.E., J. Pickering, C.H.: Eskiw, Better Living through Chemistry: Caloric Restriction (CR) and CR Mimetics Alter Genome Function to Promote Increased Health and Lifespan. Frontiers in Genetics, 2016; 7(142).

76. Mortimer, R.K., J.R. Johnston, Life span of individual yeast cells. Nature, 1959; 183(4677): p. 1751-2.

77. Lindstrom, D.L., D.E. Gottschling: The mother enrichment program: a genetic system for facile replicative life span analysis in Saccharomyces cerevisiae. Genetics, 2009; 183(2): p. 413-22, 1si-13si.

78. Cabrera, M., et al.: A simple microfluidic platform to study age-dependent protein abundance and localization changes in Saccharomyces cerevisiae. Microb Cell, 2017; 4(5): p. 169-174.

79. Park, P.U., M. McVey, L. Guarente: Separation of mother and daughter cells, in Methods in Enzymology. 2002; Academic Press. p. 468-477.

80. Powers, R.W., 3rd, et al.: Extension of chronological life span in yeast by decreased TOR pathway signaling. Genes and development, 2006; 20(2): p. 174-184.

81. Goldberg, A.A., et al.: Chemical genetic screen identifies lithocholic acid as an antiaging compound that extends yeast chronological life span in a TOR-independent manner, by modulating housekeeping longevity assurance processes. Aging (Albany NY), 2010; 2(7): p. 393-414. 
82. Liu, Y., et al.: Hormesis of glyceollin I, an induced phytoalexin from soybean, on budding yeast chronological lifespan extension. Molecules, 2014; 19(1): p. 568-80.

83. Wu, Z., et al.: Tanshinones extend chronological lifespan in budding yeast Saccharomyces cerevisiae. Appl Microbiol Biotechnol, 2014; 98(20): p. 8617-28.

84. Sprenger, J., et al.: Melatonin and Other 5-Methoxylated Indoles in Yeast : Presence in High Concentrations and Dependence on Tryptophan Availability. CYTOLOGIA, 1999; 64(2): p. 209-213.

85. Mas, A., et al.: Bioactive Compounds Derived from the Yeast Metabolism of Aromatic Amino Acids during Alcoholic Fermentation. BioMed Research International, 2014; 2014: p. 7.

86. Dai, Z., et al.: Yeast synthetic biology for high-value metabolites. FEMS Yeast Res, 2015; 15(1): p. 1-11.

87. Gibson, D.G., et al.: One-step assembly in yeast of 25 overlapping DNA fragments to form a complete synthetic Mycoplasma genitalium genome. Proc Natl Acad Sci U S A, 2008; 105(51): p. 20404-9.

88. Naesby, M., et al.: Yeast artificial chromosomes employed for random assembly of biosynthetic pathways and production of diverse compounds in Saccharomyces cerevisiae. Microb Cell Fact, 2009; 8: p. 45.

89. Jiang, H., K.V. Wood, J.A. Morgan: Metabolic engineering of the phenylpropanoid pathway in Saccharomyces cerevisiae. Applied and environmental microbiology, 2005; 71(6): p. 2962-2969.

90. Hammer, S.K., J.L. Avalos: Harnessing yeast organelles for metabolic engineering. 2017; 13(8): p. 823-832.

91. Yan, Y., A. Kohli, M.A. Koffas: Biosynthesis of natural flavanones in Saccharomyces cerevisiae. Appl Environ Microbiol, 2005; 71(9): p. 5610-3.

92. Jakociunas, T., et al.: Multiplex metabolic pathway engineering using CRISPR/Cas9 in Saccharomyces cerevisiae. Metab Eng, 2015; 28: p. 213-222.

93. Trantas, E., N. Panopoulos, F. Ververidis: Metabolic engineering of the complete pathway leading to heterologous biosynthesis of various flavonoids and stilbenoids in Saccharomyces cerevisiae. Metab Eng, 2009; 11(6): p. 355-66.

94. Becker, J.V., et al.: Metabolic engineering of Saccharomyces cerevisiae for the synthesis of the wine-related antioxidant resveratrol. FEMS Yeast Res, 2003; 4(1): p. 79-85.

95. Liang, H., et al.: Production of a bioactive unnatural ginsenoside by metabolically engineered yeasts based on a new UDP-glycosyltransferase from Bacillus subtilis. Metab Eng, 2017; 44: p. 60-69. 